\title{
The Effects of Hyperemesis Gravidarum on the Oral Glucose Tolerance Test Values and Gestational Diabetes
}

\author{
Burak Bayraktar, Meriç Balıkoğlu, Miyase Gizem Bayraktar, \\ Ahkam Goksel Kanmaz \\ Department of Obstetrics and Gynecology, University of Health Sciences \\ Tepecik Training and Research Hospital, Izmir, Turkey \\ Received March 23, 2021; Accepted October 20, 2021.
}

Key words: Hyperemesis gravidarum - Gestational diabetes - Oral glucose tolerance test - Pregnancy complications - Pregnancy outcome - Fetal macrosomia

\begin{abstract}
This study is aimed at determination whether pregnant women who develop hyperemesis gravidarum in the first trimester have a tendency to develop gestational diabetes mellitus (GDM). It is also aimed at identification of effects of hyperemesis gravidarum and GDM on prenatal and neonatal status in case they were detected together. Hyperemesis gravidarum diagnose was based on the following signs and symptoms. To diagnose GDM, first trimester fasting blood glucose measurement and subsequent blood glucose monitoring and 75-g oral glucose tolerance test (OGTT) were performed in the second trimester. A total of 949 singleton pregnant women ( 95 with and 852 without hyperemesis gravidarum) who met our criteria were included in the study. In the first trimester, plasma blood glucose and positive GDM screening were found to be significantly higher in the hyperemesis gravidarum group compared to the control group $(p=0.042$ and $p<0.001$, respectively). However, actual GDM cases were similar between both groups. The positive predictive value was significantly lower in the hyperemesis gravidarum group $(28.5 \%$ vs. $72.7 \%, p=0.003)$. In the second trimester, the prevalence of GDM was $6.6 \%$ in the hyperemesis gravidarum group and $7.3 \%$ in the control group, with no significant difference $(p=0.218)$ between-groups. In this study, hyperemesis gravidarum was found to cause changes in maternal metabolism in the first trimester of pregnancy due to limited calorie intake and fasting; in the presence of hyperemesis gravidarum, it should be known that the positive predictive value of first trimester gestational diabetes screening may decrease and the diagnosis of pseudo-GDM may increase.
\end{abstract}

Mailing Address: Burak Bayraktar, MD., Department of Obstetrics and Gynecology, University of Health Sciences Tepecik Training and Research Hospital, Guney Street, 35170 Izmir, Turkey; Phone: +90 23246969 69; Fax: +90 23245796 51; e-mail: drburakbayraktar@gmail.com 


\section{Introduction}

Nausea often refers to an uneasy sensation including an urge to vomit; vomiting can be defined as the oral excretion of stomach and duodenum contents due to the contraction of the diaphragm and other abdominal muscles (Golembiewski et al., 2005). Although vomiting is frequently preceded by nausea, it may occur without nausea or with persistent nausea (Golembiewski et al., 2005).

Nausea and vomiting are common complaints in $50-80 \%$ of pregnant women, negatively impacting their family, social, and work life (Matthews et al., 2010). Symptoms usually begin at 2-4 weeks after the last menstrual period; they peak at 9-16 weeks and disappear until 22 weeks of gestation at the latest (Lacroix et al., 2000).

Hyperemesis gravidarum is a pregnancy complication characterized by vomiting, malnutrition due to excessive vomiting, ketonemia-ketonuria, electrolyte imbalance, and, ultimately, loss of $>5 \%$ of body weight (Pollack and ACOG Committee on Practice Bulletins-Gynecology, 2003), with its frequency ranging from $0.3 \%$ to $3 \%$ (Matthews et al., 2010). Persistent symptoms continue during pregnancy in $10 \%$ of pregnant women, although they usually disappear around 22 weeks (Lacroix et al., 2000). Although spontaneous recovery is frequently observed, dehydration and ketonemia may lead to electrolyte and acid-base imbalance, which could eventually lead to hepatic and renal failure (Fairweather, 1968). The etiopathogenesis of hyperemesis gravidarum remains to be fully elucidated; however, it is associated with a number of factors such as pregnancy hormones (human chorionic gonadotropin, estradiol, progesterone), hyperthyroidism, upper gastrointestinal dysmotility, immune system dysfunction, nutritional disorders, helicobacter pylori infection, and psychological factors that may exacerbate the situation (Pollack and ACOG Committee on Practice Bulletins-Gynecology, 2003). The American College of Obstetricians and Gynecologists (ACOG) recommends early intervention to prevent the progression of nausea and vomiting during pregnancy to hyperemesis gravidarum, which negatively affects a women's family, social, and business life (Pollack and ACOG Committee on Practice Bulletins-Gynecology, 2003).

Gestational diabetes mellitus (GDM) can be defined as glucose intolerance of any degree, which is first diagnosed or first occurs during pregnancy (American Diabetes Association, 2003). Approximately $7 \%$ of all pregnancies are complicated by GDM, with this rate varying between $1 \%$ and $14 \%$ in different populations (American Diabetes Association, 2003). Compared with non-pregnant women, to maintain maternal euglycemia, the amount of insulin secreted from the pancreas due to increased insulin resistance seems to be higher among pregnant women. During pregnancy, insulin resistance is physiologically tolerated by healthy women, whereas those with diabetes mellitus (DM) or those who did not have DM before pregnancy cannot compensate for the insulin resistance; therefore, GDM may occur in them (Creasy et al., 2009). 
Due to the maternal metabolism that is impaired because of hyperemesis gravidarum and starvation, ketone formation occurs through fatty acid oxidation. In vitro study has shown that long-term fatty acid oxidation and ketone accumulation in the plasma may impair pancreatic B-cell functions and decrease insulin secretion and may increase blood glucose levels (Zhou and Grill, 1995).

Therefore, this study aimed to determine whether pregnant women who develop hyperemesis gravidarum in the first trimester have a tendency to develop GDM and aimed to identify the effects of hyperemesis gravidarum and GDM on prenatal and neonatal status in case they were detected together.

\section{Material and Methods}

The study was designed as a retrospective cohort. Pregnant women who were diagnosed with hyperemesis gravidarum between 2016 and 2019, who underwent first trimester fasting glucose measurement and were followed up using a $75-\mathrm{g}$ oral glucose tolerance test (OGTT) were selected as the study group. Pregnant women whose follow-up results could be accessed through the hospital information system, who gave birth at our hospital, and for whom newborn treatment and newborn intensive care follow-ups were provided at our hospital were included in this study. Hyperemesis gravidarum was diagnosed based on the following signs and symptoms: nausea and vomiting combined with ketone in urine made with dipstick test, $>5 \%$ of body weight loss, or severe nausea and vomiting that could limit fluid intake, even when the other condition were absent (Pollack and ACOG Committee on Practice Bulletins-Gynecology, 2003). Pregnant women aged $<18$ and $>35$ years and those with multiple pregnancies; with fetal weight $<500$ g; with known type 1 and type 2 DM; with hepatitis, gastroenteritis, pyelonephritis, or urolithiasis; and with missing findings in their records were excluded. Pregnant women without any additional pregnancy complications, whose fasting plasma glucose was measured in the first trimester and who were administered 75-g OGTT and gave birth at our hospital were included as the control group in the study.

In the protocol of our hospital, peripheral blood glucose measurements are made six times a day for pregnant women with fasting plasma glucose of $\geq 92 \mathrm{mg} / \mathrm{dl}$ in the first trimester. Based on these measurement values, gestational diabetes is diagnosed. In addition, all pregnant women are administered 75-g OGTT between the 24 and 28 weeks of gestation according to the recommendations of the International Association of Diabetes and Pregnancy Study Groups (IADPSG) (International Association of Diabetes and Pregnancy Study Groups Consensus Panel et al., 2010). To diagnose GDM, values $\geq 92 \mathrm{mg} / \mathrm{dl}$ after fasting, $180 \mathrm{mg} / \mathrm{dl}$ after the first hour, and $153 \mathrm{mg} / \mathrm{dl}$ after the second hour are used during the second 75-g OGTT as accepted by IADPSG (International Association of Diabetes and Pregnancy Study Groups Consensus Panel et al., 2010). Simultaneously, newborns weighing $\geq 4,000 \mathrm{~g}$ were considered to have macrosomia. 
Ethics committee approval for this study was obtained from the University of Health Science Tepecik Education and Research Hospital Ethics Committee and informed consent was not necessary due to the retrospective nature of this study.

Statistical analysis

The Statistical Package for the Social Sciences (SPSS) 22.0 version (IBM Corporation, Armonk, New York, US) software package was used for statistical analysis. The normally distributed variables were evaluated using Kolmogorov-Smirnov $(n>30)$ and Shapiro-Wilk $(n<30)$ tests. For parametric variables, student's $t$-test was used and data were presented as mean \pm standard deviation, whereas for non-parametric variables, Mann-Whitney $U$ test was used and data were presented as median \pm (min, max). For categorical variables between the groups, chi-square test was used and odds ratio (95\% confidence interval $[\mathrm{Cl}]$ ) was calculated. Results were considered significant at $\mathrm{p}<0.05$.

\section{Results}

A total of 947 singleton pregnant women (95 with and 852 without hyperemesis gravidarum) followed up at our hospital between 2016 and 2019 were included in the study. Their demographic information is summarized in Table 1. Although the frequency of nulliparity was higher in the hyperemesis gravidarum group, no significant difference was found between the two groups in terms of age, parity, and fetal gender. Furthermore, no significant difference was found between the groups when body mass index (BMI) was evaluated during the test.

In the first trimester, plasma fasting blood glucose and positive GDM screening were found to be significantly higher in the hyperemesis gravidarum group compared

Table 1 - Demographic features of pregnant women involved in the study

\begin{tabular}{lccc}
\hline & $\begin{array}{c}\text { Hyperemesis gravidarum } \\
\text { group (n=95) }\end{array}$ & $\begin{array}{c}\text { Control } \\
\text { group (n=852) }\end{array}$ & P-value \\
\hline Age (mean \pm SD) & $30.6 \pm 6.3$ & $30.6 \pm 6.6$ & 0.855 \\
\hline Parity $(\mathrm{n}, \%)$ & & & 0.143 \\
\hline Primipara & $35(36.8 \%)$ & $252(29.5 \%)$ & \\
Multipara & $60(63.2 \%)$ & $600(70.5 \%)$ & 0.611 \\
\hline Gender $(\mathrm{n}, \%)$ & & & \\
\hline Male & $46(44.7 \%)$ & $436(51.9 \%)$ & \\
Female & $49(55.3 \%)$ & $416(48.1 \%)$ & 0.742 \\
\hline $\begin{array}{l}\text { BMl during test } \\
\text { (mean } \pm \mathrm{SD})\left(\mathrm{kg} / \mathrm{m}^{2}\right)\end{array}$ & $25.3 \pm 4.2$ & $26.8 \pm 5.7$ & \\
\hline
\end{tabular}

$\mathrm{BMI}$ - body mass index; SD - standard deviation 


\section{Table 2 - Values of fasting plasma glucose among pregnant women and presence of gestational diabetes}

\begin{tabular}{lccr}
\hline & $\begin{array}{c}\text { Hyperemesis } \\
\text { gravidarum group } \\
(\mathbf{n = 9 5 )}\end{array}$ & $\begin{array}{c}\text { Control group } \\
\mathbf{( n = 8 5 2 )}\end{array}$ & P-value \\
\hline Positive GDM screening test (n, \%) & $14(15.7 \%)$ & $44(5.1 \%)$ & $<0.001$ \\
GDM prevalence (n, \%) & $4(4.2 \%)$ & $32(3.7 \%)$ & 0.826 \\
Positive predictive value (\%) & 28.5 & 72.7 & 0.003 \\
Fasting (median, min-max) (mg/dl) & $89.6(60-158)$ & $84.6(57-153)$ & 0.042 \\
\hline
\end{tabular}

GDM - gestational diabetes mellitus

\section{Table 3 - Values of 75-g oral glucose tolerance test among pregnant women and presence of gestational diabetes}

\begin{tabular}{lccc}
\hline & $\begin{array}{c}\text { Hyperemesis } \\
\text { gravidarum group } \\
(\mathbf{n = 9 1 )}\end{array}$ & $\begin{array}{c}\text { Control group } \\
\mathbf{( n = 8 2 0 )}\end{array}$ & P-value \\
\hline GDM prevalence $(\mathrm{n}, \%)$ & $6(6.6 \%)$ & $60(7.3 \%)$ & 0.800 \\
Fasting (median, min-max) $(\mathrm{mg} / \mathrm{dl})$ & $86.9(59-151)$ & $86(61-203)$ & 0.888 \\
Hour 1 (median, min-max) $(\mathrm{mg} / \mathrm{dl})$ & $148.6(82-328)$ & $145.6(41-315)$ & 0.749 \\
Hour 2 (median, min-max) $(\mathrm{mg} / \mathrm{dl})$ & $118.5(60-313)$ & $114.7(32-283)$ & 0.563 \\
\hline
\end{tabular}

GDM - gestational diabetes mellitus

to the control group ( $p=0.042$ and $p<0.001$, respectively). However, actual GDM cases were similar between the groups. The positive predictive value was significantly lower in the hyperemesis gravidarum group $(28.5 \%$ vs. $72.7 \%, p=0.003)$. The findings are summarized in Table 2.

In the second trimester, the prevalence of GDM was $6.6 \%$ in the hyperemesis gravidarum group and $7.3 \%$ in the control group, with no significant between-group difference $(p=0.218)$. Similarly, 75-g OGTT administered after fasting and after the first and second hours showed that there was no significant difference between the hyperemesis gravidarum group and the control group $(p=0.888, p=0.749$, and $p=0.563$, respectively). The findings are summarized in Table 3.

The effects of hyperemesis gravidarum as a secondary analysis on the outcomes in pregnant women with GDM are summarized in Table 4. There was no significant difference between the group with hyperemesis gravidarum with GDM and the group with only GDM in terms of birth week and weight. The frequency of macrosomia was similar between the groups. Moreover, the number of newborns with Apgar scores of $<7$ in the first and fifth minutes was lower in the hyperemesis gravidarum group, with no significant between-group difference. The need for neonatal intensive care unit (NICU) admission was found to be similar between the two groups. 


\section{Table 4 - Delivery outcomes in gestational diabetes mellitus with together hyperemesis gravidarum and the group with only gestational diabetes}

\begin{tabular}{lccc}
\hline & $\begin{array}{c}\text { Group with GDM } \\
\text { and hyperemesis } \\
\text { gravidarum (n=10) }\end{array}$ & $\begin{array}{c}\text { Group with } \\
\text { only GDM } \\
(\mathbf{n = 9 2 )}\end{array}$ & P-value \\
\hline Gestational age at delivery (mean \pm SD) & $38.3 \pm 1.5$ & $38.6 \pm 2.0 \mathrm{sp}$ & 0.327 \\
Preterm delivery (n, \%) & $1(10 \%)$ & $12(13.0 \%)$ & 0.784 \\
Birth weight (mean \pm SD) $(\mathrm{g})$ & $3237 \pm 587$ & $3309 \pm 437$ & 0.724 \\
Macrosomia (n, \%) & $1(10 \%)$ & $20(21.7 \%)$ & 0.383 \\
1 minutes Apgar score $<7$ (n, \%) & $1(10 \%)$ & $12(13.0 \%)$ & 0.784 \\
5 minutes Apgar score $<7(\mathrm{n}, \%)$ & $1(10 \%)$ & $10(10.9 \%)$ & 0.932 \\
NICU admission (n, \%) & $1(10 \%)$ & $10(10.9 \%)$ & 0.932 \\
\hline
\end{tabular}

GDM - gestational diabetes mellitus; NICU - neonatal intensive care unit; SD - standard deviation

\section{Discussion}

In this study, hyperemesis gravidarum was found to cause changes in maternal metabolism in the first trimester of pregnancy due to limited calorie intake and fasting; however, it did not increase the incidence of GDM.

In a retrospective analysis performed by Ohara et al. (2016), OGTT was performed in the first trimester in pregnant women with hyperemesis gravidarum, and the incidence of screening test positivity found to be higher in hyperemesis gravidarum group. However, similar to our study, the actual prevalence of gestational diabetes was similar between the groups. In addition, first trimester fasting blood glucose level values were compared between the groups in our study and it was found to be significantly higher in the hyperemesis gravidarum group. Hyperemesis gravidarum is a complication, which can be detected in $0.3-3 \%$ of pregnant women; it affects calorie intake at varying levels and may cause ketonemia due to fatty acid oxidation (Pollack and ACOG Committee on Practice Bulletins-Gynecology, 2003; Matthews et al., 2010). In the acute period; prolonged fasting, low calorie intake and ketonuria may decrease insulin secretion and increase blood glucose levels; thus, hypothetically it would reasonably increase the incidence of pseudo-GDM in this group of patients (Zhou and Grill, 1995). Also, a stress response caused by vomiting and nausea may contribute to high glycemia in the hyperemesis gravidarum group, especially if present at the time of blood glucose determination.

Both Ohara et al. (2016) and Madendag et al. (2018) investigated the prevalence of GDM in the second trimester. However, similar to us, they found no difference between the groups in terms of GDM in the 75-g OGTT test they performed between 24-28 weeks of gestation. This may be explained by the nature of hyperemesis gravidarum. Majority of pregnant women with hyperemesis gravidarum regain their health at the beginning of the second trimester; however, OGTT is still 
performed until the end of the second trimester. Therefore, the incidence of GDM is not directly affected by hyperemesis gravidarum.

In our study, all values obtained for OGTT were found to be higher in the hyperemesis gravidarum group than in the control group, but the difference was not significant. Similarly, Ohara et al. (2016) have reported higher OGTT values in the hyperemesis gravidarum group both in the first and second trimesters. However, while the fasting value was higher in the hyperemesis gravidarum group, the first- and second-hour values were higher in the control group in Madendag et al.'s (2018) study. These different results are attributable to different patient selection criteria.

The secondary purpose of this study was to investigate the outcomes of pregnancies complicated by GDM with hyperemesis gravidarum. Although some studies have shown a relationship among GDM (Hedderson, 2003; Köck et al., 2010; Dorfman et al., 2015), hyperemesis gravidarum (Tierson et al., 1986; Goodwin, 2008; Veenendaal et al., 2011; Peled et al., 2013; Vikanes et al., 2013), and preterm labour, studies on pregnancies complicated by both are limited. We compare the group with hyperemesis gravidarum and GDM with the group with only GDM. The prevalence of preterm birth and gestational age at delivery were lower in the hyperemesis gravidarum group, and macrosomia was more frequent in pregnancies complicated by only GDM, but the results were not significant. Considering the literature suggesting that hyperemesis gravidarum may negatively affect the fetal weight (Bailit, 2005; Dodds et al., 2006; Roseboom et al., 2011; Veenendaal et al., 2011), it can be predicted that hyperemesis gravidarum in association with GDM decreases the incidence of macrosomia. The prevalence of newborns Apgar score of $<7$ in the first and fifth minutes and the prevalence of admission to the neonatal intensive care unit (NICU) were lower in the hyperemesis gravidarum group; but results such as other studies were not significant (Roseboom et al., 2011; Vikanes et al., 2013).

Because genetic infrastructure, nutritional habits, and race are known to be associated with DM, our study may be deficient in diversity. Another limitation of the study is the lack of regular weight monitoring. In addition, since sample size in the group with GDM and hyperemesis gravidarum group was relatively small, the reliability of the results in this table may have been affected. As our institute is a high-volume hospital, we aimed to decrease the effect of these limitations on our results by increasing the number of patients, using strict patient selection criteria, and extending the retrospective examination period.

\section{Conclusion}

In conclusion, in the presence of hyperemesis gravidarum, it should be known that the positive predictive value of first trimester gestational diabetes screening may decrease and the diagnosis of pseudo-GDM may increase. Although the incidence of actual GDM is not affected by hyperemesis gravidarum, both physicians and patients 
need to be more knowledgeable in this regard. Therefore, international, prospective randomized studies are needed on this subject.

\section{References}

American Diabetes Association (2003) Gestational diabetes mellitus. Diabetes Care 26, S103-S105 (Suppl. 1). Bailit, J. L. (2005) Hyperemesis gravidarium: Epidemiologic findings from a large cohort. Am. J. Obstet. Gynecol. 193, 811-814.

Creasy, R. K., Resnik, R., lams, J. D. (2009) Creasy and Resnik's Maternal-fetal Medicine: Principles and Practice, $6^{\text {th }}$ Edition. Saunders/Elsevier, Philadelphia.

Dodds, L., Fell, D. B., Joseph, K. S., Allen, V. M., Butler, B. (2006) Outcomes of pregnancies complicated by hyperemesis gravidarum. Obstet. Gynecol. 107(2 Pt 1), 285-292.

Dorfman, H., Srinath, M., Rockhill, K., Hogue, C. (2015) The association between diabetes mellitus among American Indian/Alaska native populations with preterm birth in eight US states from 2004-2011. Matern. Child Health J. 19, 2419-2428.

Fairweather, D. V. I. (1968) Nausea and vomiting in pregnancy. Am. J. Obstet. Gynecol. 102, 135-175.

Golembiewski, J., Chernin, E., Chopra, T. (2005) Prevention and treatment of postoperative nausea and vomiting. Am. J. Health Syst. Pharm. 62, 1247-1260.

Goodwin, T. M. (2008) Hyperemesis gravidarum. Obstet. Gynecol. Clin. North Am. 35, 401-417.

Hedderson, M. (2003) Gestational diabetes mellitus and lesser degrees of pregnancy hyperglycemia: Association with increased risk of spontaneous preterm birth. Obstet. Gynecol. 102, 850-856.

International Association of Diabetes and Pregnancy Study Groups Consensus Panel; Metzger, B. E., Gabbe, S. G., Persson, B., Buchanan, T. A., Catalano, P. A., Damm, P., Dyer, A. R., Leiva, A. D., Hod, M., Kitzmiler, J. L., Lowe, L. P., McIntyre, H. D., Oats, J. J., Omori, Y., Schmidt, M. I. (2010) International association of diabetes and pregnancy study groups recommendations on the diagnosis and classification of hyperglycemia in pregnancy. Diabetes Care 33, 676-682.

Köck, K., Köck, F., Klein, K., Bancher-Todesca, D., Helmer, H. (2010) Diabetes mellitus and the risk of preterm birth with regard to the risk of spontaneous preterm birth. J. Matern. Fetal Neonatal Med. 23 , 1004-1008.

Lacroix, R., Eason, E., Melzack, R. (2000) Nausea and vomiting during pregnancy: A prospective study of its frequency, intensity, and patterns of change. Am. J. Obstet. Gynecol. 182, 931-937.

Madendag, Y., Sahin, E., Madendag Col, I., Eraslan, S. M., Tayyar, A. T., Ozdemir, F., Acmaz, G., Senol, V. (2018) The effect of hyperemesis gravidarum on the $75 \mathrm{~g}$ oral glucose tolerance test screening and gestational diabetes mellitus. J. Matern. Fetal Neonatal Med. 31, 1989-1992.

Matthews, A., Dowswell, T., Haas, D. M., Doyle, M., O’Mathúna, D. P. (2010) Interventions for nausea and vomiting in early pregnancy. Cochrane Database Syst. Rev. 9, CD007575.

Ohara, R., Obata-Yasuoka, M., Abe, K., Yagi, H., Hamada, H., Yoshikawa, H. (2016) Effect of hyperemesis gravidarum on gestational diabetes mellitus screening. Int. J. Gynaecol. Obstet. 132, 156-158.

Peled, Y., Melamed, N., Hiersch, L., Hadar, E., Wiznitzer, A., Yogev, Y. (2013) Pregnancy outcome in hyperemesis gravidarum - The role of fetal gender. J. Matern. Fetal Neonatal Med. 26, 1753-1757.

Pollack, A.; ACOG Committee on Practice Bulletins-Gynecology (2003) ACOG practice bulletin. Clinical management guidelines for obstetrician-gynecologists. Number 46, September 2003. (Replaces technical bulletin number 222, April 1996). Obstet. Gynecol. 102, 647-658.

Roseboom, T. J., Ravelli, A. C. J., van der Post, J. A., Painter, R. C. (2011) Maternal characteristics largely explain poor pregnancy outcome after hyperemesis gravidarum. Eur. J. Obstet. Gynecol. Reprod. Biol. 156, 56-59.

Bayraktar B.; Balıkoğlu M.; Bayraktar M. G.; Kanmaz A. G. 
Tierson, F. D., Olsen, C. L., Hook, E. B. (1986) Nausea and vomiting of pregnancy and association with pregnancy outcome. Am. J. Obstet. Gynecol. 155, 1017-1022.

Veenendaal, M., van Abeelen, A., Painter, R., van der Post, J., Roseboom, T. (2011) Consequences of hyperemesis gravidarum for offspring: A systematic review and meta-analysis. BJOG 118, 1302-1313.

Vikanes, Å. V., Støer, N. C., Magnus, P., Grjibovski, A. M. (2013) Hyperemesis gravidarum and pregnancy outcomes in the Norwegian mother and child cohort - A cohort study. BMC Pregnancy Childbirth 13, 169.

Zhou, Y. P., Grill, V. (1995) Long term exposure to fatty acids and ketones inhibits B-cell functions in human pancreatic islets of Langerhans. J. Clin. Endocrinol. Metab. 80, 1584-1590. 